\title{
Intervención del trabajador social en el bienestar socioemocional de los adultos mayores
}

\author{
Social worker intervention in the socio-emotional well-being of older adults
}

Gema Beatriz Intriago-Molina' (iD) (8), Ligia Estela Loor-Lino² (iD (8)

\author{
Cómo citar \\ Intriago-Molina, G. y Loor-Lino, L. (2021). Intervención del trabajador social en el bienestar socioemocional de los adultos \\ mayores. Socialium, 5(2), 217-236. https://doi.org/10.26490/uncp.sl.2021.5.2.924
}

${ }^{1}$ Egresada de la Carrera de Trabajo Social

Universidad Técnica de Manabí, Portoviejo, Ecuador.

gintriago8813@utm.edu.ec

${ }^{2}$ Magister en Educación y Desarrollo Social, Licenciada en Trabajo Social.

Universidad Técnica de Manabí, Portoviejo, Ecuador.

ligia.loor@utm.edu.ec

Arbitrado por pares ciegos Recibido: $24 / 04 / 2021$

Aceptado: $29 / 06 / 2021$

\section{RESUMEN}

En la actualidad el envejecimiento de la población adulto mayor es un desafío en la sociedad, esta población es altamente vulnerable en el entorno familiar y social. Al ser un adulto mayor significa ser cuidado, respetado, protegido y apoyado por la familia. El estudio tuvo como objetivo analizar la intervención del trabajador social en el bienestar socioemocional de los adultos mayores, utilizando metodología cuantitativa y cualitativa y las técnicas de entrevista y encuesta para la cual se aplicó 30 encuesta a los usuarios del Centro Gerontológico Santa Gema de Galgani de la Parroquia Abdón Calderón del cantón Portoviejo y una entrevista a la trabajadora social. Los resultados muestran que la mayoría de los adultos mayores viven con sus familiares mientras que existe un porcentaje minoritario que vive solo, pese a ello se evidencia escasa comunicación con sus familiares debido a sus responsabilidades laborales, pero cuando están en situaciones de necesidad son atendidos de manera eficiente. Se concluye que, la intervención del trabajador social está focalizada en optimizar la calidad de vida del adulto mayor, para lo cual realiza actividades que favorezcan el bienestar socioemocional de los mismos, apoyándose en métodos, técnicas e instrumentos propios de la profesión como son el índice de Barthel, la escala de Lawton y Brody y el test de mini mental, que son el punto de partida para el desarrollo de actividades que favorezcan al mejoramiento de su calidad de vida.

Palabras clave: adulto mayor; trabajo social; intervención; familiar; bienestar socioemocional.

\section{ABSTRACT}

Currently the ageing of the elderly population is a challenge in society, this population is highly vulnerable in the family and social environment. Being an elderly means being cared for, respected, protected and supported by the family. The objective of the research was to analyze the intervention of the social worker in the socio-emotional well-being of the elderly, using quantitative and qualitative methodology and interview and survey techniques a survey 30 applied to users of the Santa Gema de Galgani Gerontological Center of the Abdón Calderón Parish from Portoviejo and an interview with the social worker. The results show that most of the older adults live with their relatives while there is a minor percentage who live by themselves, despite this, there is some evidence of lack of communication with their family members due to their work responsibilities, but when they are in situations of need they are efficiently cared for, It is concluded that, the intervention of the social worker is focused on optimizing the quality of life of the elderly, they carry out activities that favor their socio-emotional well-being, relying on methods, techniques and instruments of the profession such as the Barthel index, the Lawton and Brody scale and the mini mental test, which are the starting point for the development of activities that favor the improvement of their quality of life.

Keywords: older adult; social work; intervention; family; socio-emotional wellbeing. 


\section{Introducción}

En la investigación llevada a cabo por Martínez et al. (2016) aborda el tema del envejecimiento y de la vejez supone reconocer que estamos frente a una realidad compleja en la que interactúan factores biológicos, psicológicos y sociales, la vejez entendida como un período de la vida y como parte del proceso mismo de envejecimiento, ocurre en un sujeto particular y único, por lo cual la modalidad de envejecimiento no se puede generalizar a partir de los cambios que ocurren sólo a nivel físico, pues cada sujeto interpreta éstos cambios de acuerdo a sus esquemas mentales, su estructura de personalidad, creencias, valores, los procesos de socialización a los que ha sido expuesto y al lugar que ocupa dentro de un contexto social y ecológico particular.

No obstante, la Federación Iberoamericana de Asociaciones de Personas Adultas Mayores (2014) manifiesta que para la sociedad y el Estado, a nivel individual y colectivo, los desafíos que se deben de presentar para el adulto mayor son muchos, como promover la creación de políticas públicas, generar una participación real y efectiva de los adultos mayores, modificar la representación social de la vejez y poner en evidencia las diferentes formas de envejecer, es decir los adultos mayores necesitan espacios que promuevan su participación y un envejecimiento activo y saludable.

Bajo este punto de vista para la Ley Orgánica de las Personas Adultas Mayores (2019) en el Título I Principios y Disposiciones Fundamentales, Art. 1.- Objeto. Tipifica que, el objeto de esta ley es promover, regular y garantizar la plena vigencia, difusión y ejercicio de los derechos específicos de las personas adultas mayores, en el marco del principio de atención prioritaria y especializada, expresados en la Constitución de la República, instrumentos internacionales de derechos humanos y leyes conexas, con enfoque de género, movilidad humana, generacional e intercultural.

Por consiguiente, el objetivo de esta investigación es analizar la intervención del Trabajador Social en el bienestar socioemocional de los adultos mayores del centro gerontológico "Santa Gema Galgani" debido a que la pandemia del covid-19 a afectado el estado socioemocional de los adultos mayores, por lo que se debe de implementar nuevas estrategias que permita el cuidado y seguimiento de cada adulto mayor.

\section{Población Adulta Mayor}

Se define a una persona adulta mayor cuando alcanza los de 65 años de edad, las personas de la tercera edad son parte de los grupos de atención prioritaria, al igual que las personas con discapacidad, las mujeres embarazadas, los niños, niñas y adolescentes; estos grupos son considerados en la perspectiva de sujetos de derecho. Desde esta perspectiva Fernández (2012) expresa que, "en cierto sentido todo adulto mayor, independiente de su edad, puede estar en condiciones de involucrarse socialmente, más que la edad en sí misma, son otras condicionantes las que limitan la posibilidad de participación entre las personas mayores" (p. 3). Junto con lo señalado, las personas adultas mayores son sujeto de derechos, y 
son activos en la sociedad, tienen garantías y responsabilidades para sí mismo, su familia, su entorno y sus descendientes.

El Ministerio de Inclusión Económica y Social es uno de los organismos que cumple con la función de brindar atención especial e integral al adulto mayor, estima que en Ecuador constan: 1.049.824 personas mayores de 65 años (6,5\% del territorio nacional). Aleaga (2018) en su estudio realizado en la Provincia de Carchi, menciona que "el aumento de la población de adultos mayores, ha puesto a pensar al mundo en un sinnúmero de estrategias para garantizar el bienestar de las personas en todo el ciclo de vida" (p.19). Por tal razón, el Gobierno implementó políticas públicas basadas para defender los derechos y reconocer el valor y la participación de las personas mayores se incrementa gradualmente.

El envejecimiento es un proceso natural por el que atraviesan todos, y estas personas también se denominan adultos mayores. Es así que podemos verificar que, en la Constitución de la República del Ecuador, expedida en el año 2008, conforma el marco legal, conceptual y ético que refleja una forma de convivencia ciudadana en una sociedad que respeta la dignidad de todas las personas.

Por consiguiente, en la Constitución de la República del Ecuador (2008) En la sección primera adultas y adultos mayores menciona que:

Art. 36.- Las personas adultas mayores recibirán atención prioritaria y especializada en los ámbitos público y privado, en especial en los campos de inclusión social y económica, y protección contra la violencia. Se considerarán personas adultas mayores aquellas personas que hayan cumplido los sesenta y cinco años de edad. (p. 30)

Es decir, en este sentido se muestra que las personas que tienen sesenta y cinco años o más son consideradas como personas mayores, con especial énfasis en sus derechos, indicando que deben recibir una atención prioritaria para su integración activa en la sociedad.

Asimismo, en la Constitución de la República del Ecuador (2008) expresa que:

Art. 37.- El Estado garantizará a las personas adultas mayores los siguientes derechos: 1. La atención gratuita y especializada de salud, así como el acceso gratuito a medicinas. 2. El trabajo remunerado, en función de sus capacidades, para lo cual tomará en cuenta sus limitaciones. 3. La jubilación universal. 4. Rebajas en los servicios públicos y en servicios privados de transporte y espectáculos. 5. Exenciones en el régimen tributario. 6. Exoneración del pago por costos notariales y registrales, de acuerdo con la ley. 7. El acceso a una vivienda que asegure una vida digna, con respeto a su opinión y consentimiento. (p. 30)

Por lo tanto, la estipulación apoya y protege los derechos de salud y cuidado de las personas mayores de manera integral, permitiéndoles trabajar de acuerdo a sus necesidades y condiciones, y obtener una tasa 
de descuento del $50 \%$ en toso los bienes y servicios identificados. El cumplimiento de las normas constitucionales les permite vivir con dignidad en un entorno pacifico que respeta su cultura e ideología. Hay que añadir que, la Norma Técnica para la población Adulta Mayor es regulada por el Ministerio de Inclusión Económica y Social cuya finalidad para las autoras Anchundia y Loor (2019) es:

Velar por el buen funcionamiento de los centros gerontológicos que acogen y brindan atención a las personas mayores, potenciando su bienestar en las dimensiones físicas, psicológico y social para el mejoramiento de calidad de vida. En su estándar número 19 refiere que entre el equipo de talento humano de los centros gerontológicos residenciales y diurnos estará una/un Trabajador Social con título profesional de tercer nivel, por cada 30 usuarios, que cumplirá un mínimo de 20 horas de labor semanal. (p.3)

Por consiguiente, la ley orgánica de las personas adultas mayores (2019) en el “Capítulo III de los deberes del estado, sociedad y la familia frente a las personas adultas mayores" tipifica que,

Art. 11.- Corresponsabilidad de la Familia. La familia tiene la corresponsabilidad de cuidar la integridad física, mental y emocional de las personas adultas mayores y brindarles el apoyo necesario para satisfacer su desarrollo integral, respetando sus derechos, autonomía y voluntad. Es corresponsabilidad de la familia:

a) Apoyar en el proceso para fortalecer las habilidades, competencias, destrezas y conocimientos del adulto mayor.

b) Promover entornos afectivos que contribuyan a erradicar la violencia.

c) Cubrir sus necesidades básicas: una adecuada nutrición, salud, desarrollo físico, psíquico, psicomotor, emocional y afectivo.

d) Proteger al adulto mayor de todo acto o hecho que atente o vulnere sus derechos.

e) Proporcionar al adulto mayor espacios de recreación, cultura y deporte.

f) Atender sus necesidades psicoafectivas se encuentre o no viviendo en el ámbito familiar.

g) Desarrollar y fortalecer capacidades, habilidades, destrezas y prácticas personales y familiares para el cuidado, atención y desarrollo pleno de los adultos mayores en el ámbito familiar. (p.12)

\section{Bienestar Socioemocional}

Como antecedente a este estudio y tal como lo plantean Rodríguez- Mateo et al. (2016) concuerdan que: La investigación del bienestar es especialmente importante en el caso de las personas mayores, situadas en un momento vital en el que la limitación de la vida en un sentido la evidencia 
cuantitativa es más evidente, donde es más probable que aumenten las enfermedades y se mantiene la calidad de vida restante por vivir y su desarrollo es fundamental. (p. 22)

De acuerdo a lo mencionado con anterioridad, el bienestar personal o la satisfacción con la vida es tener un envejecimiento exitoso, tomando a la salud como un concepto global, incluyendo satisfacciones, felicidad, vida en general. En este sentido, verificar la salud personal de las personas mayores podemos comprender su satisfacción y felicidad, su condición y su dinámica de vida y todo esto nos permite afrontar el proceso de envejecimiento y la realidad social de los adultos mayores.

Por otro lado, la autora Carmona-Valdés (2009) en su estudio sobre el bienestar personal en el envejecimiento exterioriza que:

El ser hombre o ser mujer no es un factor que pronostique el bienestar personal en los adultos mayores en diferentes etapas de la vida tienen diferentes aspiraciones o deseos y estas expectativas no tienen que ver nada con el género, por lo que, el logro de estos deseos irá conformando la satisfacción con la vida y satisfacción con el presente, que te hará satisfecho contigo mismo, pasado, presente, trabajo, familia y otras cosas más con lo que haces. (p. 58)

A nivel internacional unos de los instrumentos que se utiliza para medir el bienestar del adulto mayor es la escala de Lawton y Brody permite medir el grado de independencia que mantiene el anciano, dándonos información valiosa para una posible intervención. Una de las principales consecuencias del envejecimiento es la pérdida progresiva de la autonomía, el deterioro de la salud física y mental influyen en que el anciano sea cada vez más dependiente, especialmente si padece algún tipo de demencia o daño cerebral. La escala de Lawton y Brody permite detectar las principales señales de dificultad y falta de autonomía en la persona (Sanz, 2020).

A ellos se añade el hecho de que, los trabajadores sociales en Ecuador a través del MIES utilizan instrumentos para medir el bienestar del adulto mayor unas escalas es el índice de Barthel que es ampliamente utilizado para este propósito y mide la capacidad de la persona para la realización de diez actividades básicas de la vida diaria como comer bañarse, vestirse, comer y ver en qué nivel de dependencia está el adulto mayor. La escala de Lawton y Brody permite medir el grado de independencia que mantiene el anciano, dándonos información valiosa para una viable intervención, como subir y bajar escaleras, ir al baño solo, coger un bus y viajar solo o si necesita compañía de algún cuidador.

\section{Intervención del trabajador social}

La intervención en trabajo social es un término muy exclusivo para la profesión, por cuanto es entendida como una acción organizada y formulada para los trabajadores sociales, pudiendo intervenir desde tres 
ámbitos como lo es desde lo individual, familiar- grupos y comunidad, su objetivo es mejorar el obstáculo para mejorar la calidad de vida de los ciudadanos.

Bajo el punto de vista de la autora Mosquera (2017) menciona que:

El Trabajador Social ha trabajado en la restitución de los derechos de las personas Adultos Mayores, enfocándose en la falta o desvío de valores en el desequilibrio socioeconómico es un motivo importante que provoca que este grupo poblacional sea violado por la sociedad e incluso su familia según opiniones expresadas, entrevistas reales con usuarios y experiencias práctica profesional del trabajo social. (p. 70)

En una investigación realizada en la Universidad Nacional de Colombia por Falla-Ramírez (2017) afirma que "la intervención de Trabajo Social es un proceso de largo aliento, no corresponde a un ejercicio espontáneo, inmediatista, tiene unos fines y unos propósitos que trascienden en el tiempo y rebasan la perspectiva asistencial y de la caridad" (p. 129).

No obstante, Rodríguez-Montañez y Manzanares-Méndez (2003) indican que la intervención de Trabajo Social con adultos mayores tiene como eje transversal:

La idea de que cada persona debe tener la oportunidad de desarrollar su potencial y de vivir una vida satisfactoria; sin embargo, se debe recordar que dentro del trabajo con adultos mayores se requieren distintos niveles de intervención, además, se debe considerar que la población de adulto mayores no es un grupo homogéneo sino un grupo heterogéneo. (p. 122)

Hay que señalar, que el proceso de envejecimiento difiere de acuerdo a las condiciones sociales, por lo tanto, es necesario seguir haciendo recomendaciones que han permitidos formas y espacios alternativos mejorando las condiciones de vida, con la intervención de programas y proyectos, los adultos mayores son individuaos activos en proceso de inclusión con la familia y la sociedad.

Bajo este punto de vista, "los Centros del Adulto Mayor son espacios de encuentro generacional orientados a mejorar el proceso del envejecimiento, mediante el desarrollo de programas de integración familiar, intergeneracional, socioculturales, recreativos, productivos y de estilos de vida para un envejecimiento activo" (Seguro Social de Salud, 2021, párr.1).

En esta situación permite afirmar que, los trabajadores sociales realizan su intervención por medio de la Misión Mis Mejores Años donde se proyecta mejorar la calidad de vida de las personas mayores de 65 años en situación de pobreza extrema, mediante el acceso progresivo al ejercicio de sus derechos, al bienestar y las oportunidades de desarrollo, a través de políticas públicas equitativas, coordinadas y ejecutadas con eficiencia, a ello hay que añadir que, existen alrededor de 208.000 personas adultas mayores en situación de pobreza (Ministerio de Inclusión Económica y Social, 2018). 
De esta forma se puede mencionar que, "Mis mejores años", es una propuesta que realizó el gobierno ecuatoriano, donde el plan se basa en un enfoque de envejecimiento activo y saludable y tiene como objetivo mejorar la calidad de vida de las personas mayores de 65 años, especialmente en aquellas que están en situación de vulnerabilidad. También tiene como objetivo reconocer que las personas mayores son sujetos de derechos y que son los principales actores en el desarrollo y la sociedad de las personas mayores. Por ello, sus prioridades incluyen tomar acciones concretas para eliminar los mitos que obstaculizan el pleno desarrollo de las personas en esta etapa.

Las funciones que desempeña y las tareas principales del trabajador social dentro de la residencia geriátrica, según Lares Comunidad Valenciana (2018) son:

- Planificación y organización del trabajo social que hay dentro del centro, mediante la programación de objetivos realizada previamente por la dirección.

- Realizar una valoración personal y familiar de cada residente.

- Realizar los informes de cada adulto mayor que permanezca en el centro e ir actualizándolo cada cierto tiempo, o cada vez que sea necesario.

- Controlar que los residentes del centro cobren debidamente su pensión y administrar bajo la supervisión de la dirección del centro la pensión de los residentes que lo requieran.

- El trabajador social, se encargará de la comunicación del ingreso de un residente en un centro y entre las entidades que sean necesarias.

- Se encargará de todas las gestiones que sean necesarias, así como de recopilar toda la documentación del adulto mayor, así como su historial clínico, para tener toda su información y seguir la evolución del mismo.

- En cada momento se encargará de asesorar y orientar al residente o sus familiares informándoles de sus derechos, además de solicitar los derechos que les correspondan.

- Una vez el adulto mayor vaya a ingresar en una residencia, el trabajador social, procederá a realizarle una entrevista con el fin de realizar una valoración del mismo para preparar y facilitar en ingreso en el centro.

- Ayudará a fomentar la integración y la participación del residente dentro del centro y ayudar a la relación entre la familia y su entorno para que no se produzca un alejamiento.

- Realizar con el resto del equipo y profesionales del centro un plan de atención personalizada a cada residente, de esta forma cada anciano estará atendido de la mejor forma posible. Así como realizar el plan 
de actividades que debe realizar cada paciente para poder mejorar dentro de lo posible su estado de salud.

- Junto con la dirección del centro, el trabajador social se encargará de gestionar las sugerencias y reclamaciones para mejorar el nivel de calidad del centro.

- El trabajador social formará parte del equipo interdisciplinar o multidisciplinar y representará la atención psicosocial del residente y la familia en todos sus aspectos.

- Fomentará la personalización e intimidad del residente en el centro, mirando siempre por la dignidad del adulto mayor.

\section{COVID-19 frente a la situación socioemocional en los adultos mayores}

En las investigaciones dadas por las Naciones Unidas (2020) expresaron que:

Algunas personas mayores han de hacer frente a vulnerabilidades adicionales en estos momentos. La propagación de la COVID-19 en las residencias e instituciones para las personas de edad está teniendo un efecto devastador en las vidas de estas, y se han recibido noticias inquietantes de casos de descuido o maltrato. Las personas de edad que pasan la cuarentena o el confinamiento con sus familiares o cuidadores pueden también estar expuestas a un riesgo mayor de sufrir violencia, malos tratos y descuido. (p. 3)

No solo las condiciones de salud y el envejecimiento ponen en peligro a las personas mayores, la soledad como emoción y el aislamiento como condición estructural, en la que viven muchas de ellas, juegan un papel importante frente a su capacidad de responder a las enfermedades, sobre todo, a una tan contagiosa y a la que son tan vulnerables como la COVID-19. Dejar de trabajar el que aún lo hacía, no asistir a los círculos de abuelos, a sus prácticas religiosas, no visitar a las amistades, a sus familiares, ni a los vecinos, no poder realizar compras de ninguna clase (Callís-Fernández et al 2020).

Las personas adultas mayores son un grupo especialmente importante, donde se ha llevado a la implementación de medidas preventivas más restrictivas de confinamiento sin embargo, estas medidas de distanciamiento social pueden inducir a efectos negativos sobre la salud de los mayores en términos de inactividad física, deterioro cognitivo, soledad o afectación del estado emocional, porque no van a poder realizar las actividades que ellos estaban acostumbrados hacer y al no ver sus familiares con la normalidad que ellos los veían y los podían visitar. 


\section{Método}

Tipo de estudio. Se trata de una investigación no experimental de tipo descriptivo, que de acuerdo al autor Tamayo (1999) permite describir las características sistemáticas de una situación (p.44), además se empleó el enfoque cualitativo y cuantitativo, el cual Guerrero (2016), indica que el enfoque cualitativo es utilizado generalmente en el análisis de las Ciencias Sociales siendo un proceso metodológico de datos cualitativos (p.2), también se empleó el enfoque cuantitativo, Hernández, et al., (2014) refiere que "este enfoque utiliza la recolección de datos para probar hipótesis con base en la medición y en análisis estadísticos"(p.4).

Población y muestra. La población objeto de la investigación era de 180 adultos mayores, por lo cual la muestra fue aleatoria intencional constituyéndose por 30 adultos mayores del centro gerontológico Santa Gema de Galgani perteneciente a la parroquia Abdón Calderón del cantón Portoviejo. Asimismo, se entrevistó a la trabajadora social que labora en el centro gerontológico Santa Gema de Galgani, para conocer los instrumentos, que desempeña un trabajador social en la intervención de los adultos mayores en el bienestar socioemocional.

Instrumentos de recolección de datos. Se utilizó el instrumento de guía que para León (2002) "permite realizar un trabajo reflexivo para la organización de los temas posibles que se abordaran en la entrevista" (p.180), aplicándose una entrevista de 6 ítems a la trabajadora social del Centro Gerontológico Santa Gema De Galgani. Asimismo, se empleó el instrumento de cuestionario, Anguita (2003) expresa que, "es un formulario que contienen las preguntas que son dirigidas a los sujetos objetos de estudio" (p.143), de tal manera se utilizó un cuestionario de 6 ítems que fue dirigido a los adultos mayores de este centro. Los instrumentos fueron validados y confiables porque se realizó la validez de contenido de las variables de estudio.

Procedimientos de la recopilación de datos. El proceso se llevó a cabo presencialmente con cada uno de los adultos mayores a quienes se les explico el objetivo del estudio, este formulario contenía las preguntas de la guía de encuesta. Posterior a ello se empleó la técnica de entrevista que según González (1997) expresa que" es un procedimiento científico para la recolección de datos, que hace referencia al proceso de interacción entre dos roles bien diferenciados que fluyen en su comunicación" (p.154). Es por tal motivo que se procedió a aplicar una entrevista con preguntas abierta a la trabajadora social de manera online, este instrumento fue de gran utilidad porque permitió conocer la importancia de la intervención del trabajador social en el bienestar socioemocional de los adultos mayores.

Aspectos éticos. Los participantes que fueron seleccionados para este estudio se les mencionó en primera instancia que sus respuestas iban hacer completamente anónimas, protegiendo su integridad y teniendo 
en cuanta la invasión a su privacidad y confidencialidad a los datos proporcionados, utilizando tal información exclusivamente para fines académicos.

Análisis de datos. El análisis de los datos se lo realizó en base a la obtención de la información contestada por los participantes durante el proceso de recolección de datos, siendo necesario la utilización del programa de Microsoft Excel, en donde se tabularon las preguntas para la obtención de los datos estadístico y tablas estadísticas.

\section{Resultado}

Tabla 1

¿Quién vive con usted?

\begin{tabular}{lcc}
\hline & Frecuencia & Porcentaje \\
\hline Cónyuge & 10 & $33 \%$ \\
Hijos & 15 & $50 \%$ \\
Nietos & 2 & $7 \%$ \\
Otros familiares & 2 & $7 \%$ \\
Solo & 1 & $3 \%$ \\
Total & 30 & $100 \%$ \\
\hline
\end{tabular}

Nota. Adultos mayores del Centro Gerontológico Santa Gema De Galgani.

Es importante destacar que las pregunta se les realizó a los adultos mayores, y se pudo evidenciar que el $50 \%$ de los adultos mayores viven con sus hijos, el $33 \%$ vive con su cónyuge, el $7 \%$ vive con los nietos, el $7 \%$ vive con otros familiares, donde ellos supieron manifestar que se quedaban con cuidadores ya que sus hijos trabajaban y el $3 \%$ vive solo en sus hogares.

Tabla 2

¿Se siente algo descontento por lo poco o mucho que ve a sus familiares?

\begin{tabular}{lcc}
\hline & Frecuencia & Porcentaje \\
\hline $\begin{array}{l}\text { Los veo tan frecuente } \\
\text { como deseo }\end{array}$ & 10 & $33 \%$ \\
$\begin{array}{l}\text { Descontento por lo } \\
\text { poco que lo veo }\end{array}$ & 20 & $67 \%$ \\
Total & 30 & $100 \%$ \\
\hline
\end{tabular}

Nota. Adultos mayores del Centro Gerontológico Santa Gema De Galgani.

Los adultos mayores del Centro Gerontológico Santa Gema De Galgani, manifestaron que el $67 \%$ se siente descontento por lo poco que ve a los familiares, ya sea por trabajo o por problemas familiares que han 
tenido en el pasado; el $33 \%$ los ve tan frecuente como ellos desean ya que entienden que es por cuestión de trabajo o por precaución al contagio por la pandemia del covid-19.

\section{Tabla 3}

¿Por qué cree usted que los familiares se alejan de los adultos mayores?

\begin{tabular}{lcc}
\hline & Frecuencia & Porcentaje \\
\hline Falta de comunicación & 18 & $60 \%$ \\
Problemas familiares & 10 & $33 \%$ \\
Otros & 2 & $7 \%$ \\
Total & 30 & $100 \%$ \\
\hline
\end{tabular}

Nota. Adultos mayores del Centro Gerontológico Santa Gema De Galgani.

Podemos evidenciar que el $60 \%$ de los adultos mayores expresan, que los familiares se alejan de ellos por la falta de comunicación, donde incluso ellos se sienten un poco culpables porque desde pequeños siempre se decía que el mayor era el que tenía la razón y donde ellos piensan que pudieron ser más expresivos con los hijos desde pequeño, ellos dicen que esa puede ser una de las causas que ahora tiene sus efectos en la falta de la comunicación; el 33 \% manifiesta que es por problemas familiares que han tenido en el pasado y en la actualidad les sigue afectando; el $7 \%$ concuerda que es por trabajo y por motivos de la pandemia que los hijos evitan visitarlos, pero indican que están en contacto con ellos ya sea por WhatsApp o mediante llamadas.

\section{Tabla 4}

¿Tiene la posibilidad de hablar con alguien de sus problemas personales o familiares?

\begin{tabular}{ccc}
\hline & Frecuencia & Porcentaje \\
\hline Si & 29 & $97 \%$ \\
No & 1 & $3 \%$ \\
Total & 30 & $100 \%$ \\
\hline
\end{tabular}

Nota. Adultos mayores del Centro Gerontológico Santa Gema De Galgani.

Hay que señalar que el $97 \%$ de los adultos mayores mencionaron que si tienen con quien hablar de sus problemas personales o familiares ya que, si cuentan con el apoyo de la familia, en estas circunstancias por el covid-19, ellos están en contacto mediante llamadas, y el 3 \% expresa que no tiene con quien poder desahogar de sus problemas personales o familiares ya que vive solo y la familia no les toma mucha importancia a sus necesidades. 
Tabla 5

¿Cuándo su familia se reúne, usted participa de todas las actividades?

\begin{tabular}{ccc}
\hline & Frecuencia & Porcentaje \\
\hline Si & 28 & $93 \%$ \\
No & 2 & $7 \%$ \\
Total & 30 & $100 \%$ \\
\hline
\end{tabular}

Nota. Adultos mayores del Centro Gerontológico Santa Gema De Galgani.

Los adultos mayores indicaron que el $93 \%$ si se reúne con su familia y participan de todas las actividades, ellos se sienten con la autoestima elevado porque si los integran y además tiene diálogos en las pequeñas reuniones que hacen y expresan que eso los hace sentir muy bien; el $7 \%$ menciona que no los integran por problemas familiares que tuvieron en el pasado o por motivos de enfermedad.

Tabla 6

¿Recibe ayuda cuando está enfermo?

\begin{tabular}{lcc}
\hline & Frecuencia & Porcentaje \\
\hline Si & 28 & $93 \%$ \\
No & 2 & $7 \%$ \\
Total & 30 & $100 \%$
\end{tabular}

Nota. Adultos mayores del Centro Gerontológico Santa Gema De Galgani.

Mencionaron que el 93 \% si reciben ayuda de algún familiar cuando ellos están enfermos y el 7 \% expresaron que no reciben ayuda ya sea porque viven solo o por problemas que han tenido en el pasado y eso sigue afectado hasta la actualidad y por eso tiene que asistir solos o decirles algún vecino que los acompañen a los centros de salud.

\section{Entrevista realizada a la trabajadora social del Centro Gerontológico Santa Gema De Galgani.}

Debido a la Pandemia por COVID-19, se realizó una entrevista online vía Zoom Video Communications a la trabajadora social del centro gerontológico Santa Gema De Galgani, para analizar su Intervención en el bienestar socioemocional de los adultos mayores y al preguntarle ¿Cuál es el rol del trabajador social en el centro gerontológico Santa Gema De Galgani? Ella expresó que:

El rol principal del Trabajador Social es potenciar en el adulto mayor a mantener niveles óptimos de su funcionamiento a pesar de sus limitaciones. La acción del profesional puede ir dirigida al 
individuo, su familia o su comunidad. Por qué cada adulto mayor se desenvuelve en un mundo diferente.

De la misma forma al preguntarle ¿Cuáles son las funciones que usted desarrolla en el centro gerontológico Santa Gema De Galgani? Refiriere que es, la función directa y las funciones de atención indirecta.

Dentro de la intervención directa esta la función de prevención, misma que se basa en la detección del problema, pues no es lo mismo que hacer diagnóstico, pero se desprende de ahí porque es el punto de partida para realizar acciones destinadas a prevenir problemas sociales, otra función es la promoción como trabajadores sociales somos promotores del desarrollo de las capacidades de las personas mayores del centro gerontológico Santa Gema de Galgani, la asistencial que permite que una vez promocionados todas estas capacidades se trabaje en pos de aumentar y fortalecer estas capacidades, la función rehabilitadora es donde re insertamos a las personas mayores, no se puede discriminar pues todos tienen diferentes capacidades, finalmente esta la función de orientación e informativa en donde el Trabajador Social asesora al usuario en cuanto temas de interés, procedimientos y cualquier tipo de requerimientos que estos tengan.

Del mismo modo en las funciones de atención indirecta se trabaja en la coordinación interinstitucional para la consecución de recursos, en caso de no tener cédula identidad se coordina con el registro civil, entre otros, el trabajo comunitario nos permite potenciar los recursos de la comunidad, la documentación es parte vital es la intervención ya que todo trabajador social debe de tener los datos del adulto mayor que se dan a través de una ficha social de un diagnóstico o un informe social, la planificación y evaluación son funciones que nos permiten planear las actividades que vamos a realizar con los adultos mayores y evaluar los resultados de las mismas, otra función de sumo interés para el trabajador social es la de formación enfoca directamente porque hay que estar en constante capacitación y obtener nuevos conocimientos, para así poder compartirlos con ellos. Cabe recalcar que la cuando se planifica a nivel macro se involucra a los adultos mayores, directivos y personal del centro en general.

Acerca de ¿Cuáles son los modelos de intervención que usted aplica en la atención de los adultos mayores? Enunció que:

Los modelos de intervención que utilizó, es el sistémico, crisis y gestión en caso ya sea para intervenir con la población adulta mayor o con la familia, mismos que permiten intervenir y desarrollar el diagnóstico para así poder establecer las necesidades y problemas que se han detectado y luego de esto se programa y se planifica que hacer con el adulto mayor en base a su 
diagnóstico y prontamente viene lo que es la ejecución, y que todo se debe de centrar en los objetivos que se deben alcanzar, luego de la ejecución se debe de realizar la evaluación que se centra en la eficacia de la intervención del Trabajador Social y cuáles fueron los recursos que se desarrollaron en el transcurso de este proceso.

Acto seguido se le preguntó ¿Cuáles son los instrumentos que usted solicita para medir el estado socioemocional en los adultos mayores? Manifestó que:

Como Trabajadora Social tengo expedientes, carpetas que manejo como la historia clínica, copias de cédula de la persona adulta mayor y del cuidador en caso de necesitar que se dan por enfermedad, se aplica la ficha social, se elabora el respectivo informe social, luego de esto utilizamos unas escalas como el índice de Barthel que es un instrumento ampliamente utilizado para este propósito y mide la capacidad de la persona para la realización de diez actividades básicas de la vida diaria como comer, bañarse, vestirse, y ver en qué nivel de dependencia está el adulto mayor. Luego la escala de Lawton y Brody permite medir el grado de independencia que mantiene el anciano, dándonos información valiosa para una posible intervención. En esta escala es medir el grado de dependencia como subir y bajar escaleras, ir al baño solo, coger un bus y viajar solo o si necesita compañía de algún cuidador. Luego El test conocido como Mini-Mental es la prueba de cribado o despistaje cognitivo más utilizada para evaluar la sospecha de síntomas compatibles con deterioro cognitivo o demencia del adulto mayor como sumar, restar, lectura comprensiva luego de aplicar todos estos instrumentos aplico la Escala de Depresión Geriátrica de Yesavage es un instrumento ampliamente utilizado para tamizaje de depresión y en la versión breve se considera un resultado normal los valores de 0 a 5 puntos.

De la misma forma al preguntarle ¿Cuáles son las actividades que usted realiza para promover el bienestar socioemocional de los adultos mayores? alegó que:

Las actividades son muchas e involucran a las familias de los adultos mayores, se trabaja el ámbito familiar, artístico, cultural, deportivos, motriz y destreza, mismas que son muy importante porque permite el estímulo de la creatividad y contribuyen al bienestar y autoestima de los participantes.

En la finalización de la entrevista se le preguntó ¿Cómo ha afectado el covid-19 en estado socioemocional en los adultos mayores? argumentó que:

Mucho, entre ellos el deterioro físico, cognitivo, intelectual y emocional de los adultos mayores, y para ellos el simple hecho de ir envejeciendo e ir perdiendo sus movilización es una parte muy dura, más aun que ellos ahora se encuentran encerrados en su casa por precaución, situación que les ha resultado difícil porque han estado acostumbrado a asistir al centro de adulto mayor Santa 
Gema De Galgani, ya que no pueden compartir tiempo con sus compañeros y la compañía de la Trabajadora Social.

\section{Discusión}

Es importante destacar que el adulto mayor vive en el núcleo familiar y bajo este punto de vista MoralesMartínez (2014) expresa que,

La persona adulta mayor debe, idealmente, permanecer en su propio domicilio, donde ha vivido durante toda su vida, no hay mejor lugar que su habitación, donde todo le es familiar, está llena de recuerdos sentimentales y personales, los cuales constituyen su gran todo. (párr.2)

Por consiguiente, los adultos mayores del Centro Gerontológico Santa Gema De Galgani expresaron que, se siente un poco descontento por lo poco que ve a los familiares, ya sea por trabajo o por problemas familiares y pasando a los estudios de Yanes-García (2011) se puede decir que, "Una de las problemáticas que más afecta a los ancianos en nuestro medio, es la convivencia familiar, la cual generalmente es generadora de conflictos intergeneracionales dados por dificultades en la comunicación" (p.140).

Se pudo evidenciar que los familiares se alejan de los adultos mayores porque no existe una buena comunicación y desde esta perspectiva Negrete (2015) alega que, debido a las circunstancias propias en la tercera edad, ellos necesitan una familia que los apoye y sostenga, unos de los beneficios del acompañamiento de los familiares en el cuidado de los adultos mayores es que se les reconozcan que ellos tienen fortalezas y habilidades, mediante alguna nueva actividad que sea de su interés que los mantenga física y mentalmente activos.

Los adultos mayores en su gran mayoría supieron manifestar que si participan de todas las actividades que realizan sus familiares, y en la investigación llevada a cabo por Valdez-Huirache y Álvarez -Bocanegra, (2018) Indica que, los adultos mayores tienen buena calidad de vida, y en su mayoría cuenta con apoyo familiar. Hay una asociación estadísticamente significativa entre calidad de vida y apoyo familiar: A mayor apoyo familiar mayor calidad de vida.

Desde el punto de vista de Suárez y Gross (2019) es importante, el apoyo familiar brindado al paciente adulto mayor impacta positivamente en su condición física, en su bienestar psicológico y en sus relaciones sociales, este puede ser considerado como una vía necesaria emocionalmente importante y coherente, pues posibilita la estimulación cognitiva precoz del paciente adulto mayor. Donde los adultos mayores supieron corroborar que ellos si cuentan con ayuda cuando están enfermos. 
Por consiguiente, Filardo (2011) expresa que, "el objetivo de la intervención gerontológica en trabajo social es conocer y transformar la realidad del adulto mayor" (p.213). Hay que añadir, además que, Melara y Quijada (2012) exterioriza que, "el trabajador social tiene que brindar una atención integral y que responda a las necesidades del adulto mayor" (p.23).

\section{Conclusión}

La intervención del trabajador social centrada en la atención a los adultos mayores se basa principalmente en la utilización de un conjunto de herramientas fundamentales para medir el nivel de bienestar socioemocional, mediante la aplicación de los instrumentos como el índice de Barthel, la escala de Lawton y Brody, el test de mini mental y la Escala de Depresión Geriátrica de Yesavage, que son el punto de partida para el desarrollo de actividades que favorezcan al mejoramiento de su calidad de vida. No obstante, las funciones que desempeña el profesional del área social dentro del bienestar socioemocional de los adultos mayores, son orientar, dirigir, promocionar, organizar, entre otras que complementa la intervención en este campo de acción.

La investigación, planificación, ejecución y evaluación son fases del proceso metodológico que aplica la trabajadora social en su intervención profesional con las personas de la tercera edad y familiares, donde se ha visto afectado el entorno socioemocional de los adultos mayores y por tal motivo se ha tenido que implementar nuevas estrategias virtuales que permitan atender y dar seguimiento a cada uno de los usuarios del Centro Gerontológico Santa Gema de Galgani. La mayoría de los adultos mayores viven con su familia, a los que ven muy poco por situaciones laborales, no obstante, existe un porcentaje minoritario que vive solo, por lo tanto, la intervención del trabajador social es lograr que el adulto mayor tenga niveles óptimos en el bienestar socioemocional. Además, se pudo evidenciar que los adultos mayores del Centro Gerontológico Santa Gema de Galgani se sienten amados, queridos y respetados, situación que favorece a que ellos tengan un buen bienestar socioemocional.

\section{Referencias}

Aleaga, A. (2018). Análisis de la situación del adulto mayor en Quito. Caso: Hogar de Ancianos Santa Catalina Labouré, período 2017 [Tesis de maestría, Universidad Andina Simón Bolívar]. Repositorio institucional UASB. https://repositorio.uasb.edu.ec/handle/10644/6465

Anguita, J. (2003). La encuesta como técnica de investigación. Elaboración de cuestionarios y tratamiento estadístico de los datos (I). Aten Primaria 31(8),527-38. https://core.ac.uk/download/pdf/82245762.pdf 
Anchundia, K. y Loor, L. (2019) Situación socioeconómica de los usuarios de los centros gerontológicos públicos y privados del Cantón Portoviejo, 2019, Revista Caribeña de Ciencias Sociales 1-12. https://www.eumed.net/rev/caribe/2019/10/usuarios-centros-gerontologicos.pdf

Callís-Fernández, S., Guarton-Ortiz, O., Cruz-Sánchez, V., y de-Armas-Ferrera, A. (2021). Estados afectivos emocionales en adultos mayores solos en aislamiento social durante la pandemia COVID19. Revista ElectróNica Dr. Zoilo E. Marinello Vidaurreta, 46(1). http://revzoilomarinello.sld.cu/index.php/zmv/article/view/2491

Carmona-Valdés, S. (2009). El bienestar personal en el envejecimiento. Iberóforum. Revista de Ciencias Sociales de la Universidad Iberoamericana, 4(7), 48-65. https://www.redalyc.org/pdf/2110/211014858003.pdf

Constitución de la República de Ecuador [Const.]. Artículo 36. [Título I]. Registro Oficial 449 de 20 de octubre de 2008 (Ecuador). https://bit.ly/2B93igl

Constitución de la República de Ecuador [Const.]. Artículo 37. [Título I]. Registro Oficial 449 de 20 de octubre de 2008 (Ecuador). https://bit.ly/2B93igl

Falla, U. (2017). La intencionalidad de la intervención del Trabajo Social. Trabajo Social 1 (9) 123-135. http://www.scielo.org.co/pdf/traso/n19/2256-5493-traso-19-123.pdf

Federación Iberoamericana de Asociaciones de Personas Adultas Mayores [FIAPAM]. (2014, 23 de marzo). Los desafíos del envejecimiento en Ecuador. https://fiapam.org/los-desafios-delenvejecimiento-enecuador/\#: :text=En\%20Ecuador\%2C\%20las\%20cifras\%20difundidas,principalmente\%20en\%20S ierra\%20y\%20Costa.

Fernández, B. (2012). Participación social entre los adultos mayores chilenos. Revista Trabajo Social 83, 61-68. http://revistatrabajosocial.uc.cl/index.php/RTS/article/view/19059/15679

Filardo Llamas, C. (2011). Trabajo Social para la Tercera Edad. Dialnet, 213-216.

Guerrero, M. (2016). La Investigación Cualitativa. INNOVA Research Journal, 1(2), 1-9. https://doi.org/10.33890/innova.v1.n2.2016.7

González, M. (1997). Metodología de la investigación social. Técnicas de recolección de datos. Aguaclara. 
Hernández, R., Fernández, C. y Baptista, M. (2014). Metodología de la investigación (6ta ed.). McGrawHill.

Lares Comunidad Valenciana[LARESCV]. (2018). Trabajador social, ¿Qué importancia tiene en la vida del anciano? https://www.larescvalenciana.org/trabajador-social-importancia-vidaanciano/\#: :text=El\%20trabajador\%20social\%20formar\%C3\%A1\%20parte,la\%20dignidad\%20del \%20adulto\%20mayor.

Ley orgánica de las personas adultas mayores. (27 de Marzo de 2019). Asamblea Nacional. Art. 1. https://www.gob.ec/sites/default/files/regulations/2019-

06/Documento_\%20LEY\%20ORGANICA\%20DE\%20LAS\%2OPERSONAS\%20ADULTAS\%20MAYORE S.pdf

Ley orgánica de las personas adultas mayores. (27 de Marzo de 2019). Asamblea Nacional. Art. 11. https://www.gob.ec/sites/default/files/regulations/2019-

06/Documento_\%20LEY\%20ORGANICA\%20DE\%20LAS\%20PERSONAS\%20ADULTAS\%20MAYORE S.pdf

León, A. (2002). Estrategias Para el Desarrollo de la Comunicación Profesional. Editorial Limusa. https://books.google.com.ec/books?id=2yXjdKrQh$4 C \&$ printsec=frontcover $\&$ hl=es $\&$ source=gbs_ge_summary_r $\&$ cad $=0$

Martinez, H., Mitchell, M. y Aguirre, C. (2016). Salud del Adulto Mayor - Gerontología y Geriatría. Manual de Medicina Preventiva y Social I [Archivo PDF]. http://preventivaysocial.webs.fcm.unc.edu.ar/files/2014/04/Unidad-5-Salud-Adulto-Mayor-V2013.pdf

Melara, M. y Quijada, J. (2012). Intervención del Trabajo Social con los Adultos Mayores en Instituciones Públicas y Privadas del Área de San Salvador (2011-2012) [Tesis de pregrado, Universidad de el Salvador]. Repositorio institucional UES. http://ri.ues.edu.sv/id/eprint/2606/1/Intervenci\%C3\%B3n_del_trabajo_social_con_los_adultos_ mayores.pdf

Méndez, A. y Astudillo M. (2008). La investigación en la era de la información: guía para realizar la bibliografía y fichas de trabajo. Trillas. http://www.economia.unam.mx/academia/inae/pdf/inae1/u1l5.pdf

Ministerio de Inclusión Económica y Social. (2018, 7 de noviembre). Misión "Mis Mejores Años" promueve la inclusión de adultos mayores en la comunidad. 
https://www.inclusion.gob.ec/mision-mis-mejores-anos-promueve-la-inclusion-de-adultosmayores-en-la-comunidad/

Morales Martínez, F. (2014, octubre 1). ¿Dónde deben vivir los adultos mayores? La Nacion. https://www.nacion.com/opinion/foros/donde-deben-vivir-los-adultosmayores/YOKUVOQJZREPLKK5FZYEVB2DHA/story/\#: :text=Para\%20la\%20interrogante\%20de\%2 Od\%C3\%B3nde,domicilio\%20o\%20en\%20su\%20comunidad.\&text=La\%20persona\%20adulta\%20 mayor\%20debe,vivido\%20durante

Mosquera, L. (2017). Impacto de la intervención del trabajo social en el grupo de adultos mayores de la parroquia rural de san miguel de perucho periodo octubre 2015-febrero 2016 [Tesis de grado Universidad Central del Ecuador]. Repositorio institucional UCE. http://www.dspace.uce.edu.ec/bitstream/25000/13427/1/T-UCE-0017-TS047-2017.pdf

Naciones Unidas. (2020, mayo). Informe de políticas: Los efectos de la COVID-19 en las personas de edad. https://www.un.org/sites/un2.un.org/files/old_persons_spanish.pdf

Negrete, D. (2015, 23 de enero). 6 beneficios del apoyo familiar en la salud emocional del adulto mayor. Hogar del corazón de Jesús Junta de Beneficiencia de Guayaquil. https://hogarcorazondejesus.org.ec/blog/item/20003-beneficios-apoyo-familiar-aporta-saludemocional-adulto-mayor

Rodríguez-Mateo, H., Díaz-Negrín, M., Torrecillas, A., Luján, I. y Rodríguez-Rodríguez, R.(2016) Variables socioemocionales y bienestar psicológico en personas mayores. Revista Internacional de Psicología del Desarrollo y la Educación, 1(2), 21-36. https://www.redalyc.org/pdf/3498/349851778003.pdf

Rodríguez, y ., Manzanares, A. (2003). Intervención de trabajo social con adultos mayores. Revista de Trabajo Social 5, 119-130. https://dialnet.unirioja.es/servlet/articulo?codigo=4388614

Sanz, E. (2020, enero 20). La escala de Lawton y Brody para evaluar la autonomía en el anciano. La mente es maravillosa. https://lamenteesmaravillosa.com/la-escala-de-lawton-y-brody-paraevaluar-la-autonomia-en-el-anciano/

Seguro Social de Salud. (2021, 17 de febrero). ¿Que es un Centro del Adulto Mayor - CAM? http://www.essalud.gob.pe/adulto-mayor/

Suárez, L. y Gross, R. (2019). Estimulación cognitiva y apoyo familiar hacia adulto mayor con deterioro cognitivo. Revista Información Científica, 98(1), 88-97. http://scielo.sld.cu/scielo.php?script=sci_arttext\&pid=S1028- 
99332019000100088\#: :text=La\%20estimulaci\%C3\%B3n\%20temprana\%20de\%20los,las\%20parc ialmente\%20deterioradas\%20por\%20desuso.

Tamayo, M. (1999). Aprender a investigar. Instituto Colombiano Para El Fomento De La Educación Superior ICFES [Archivo PDF].

https://academia.utp.edu.co/grupobasicoclinicayaplicadas/files/2013/06/2.-LaInvestigaci\%c3\%b3n-APRENDER-A-INVESTIGAR-ICFES.pdf

Valdez-Huirache, M. y Álvarez-Bocanegra, C. (2018). Calidad de vida y apoyo familiar en adultos mayores adscritos a una unidad de medicina familiar. Horizonte sanitario, 17(2), 113-

121. https://doi.org/10.19136/hs.a17n2.1988

Yanes, M. (2011). Consejos y propuestas para un envejecimiento satisfactorio. MediSur, 9(4), 407-417. http://scielo.sld.cu/scielo.php?script=sci_arttext\&pid=S1727-897X2011000400019

\section{Contribución de los autores}

GBIM: Conceptualización, análisis formal, investigación, redacción: borrador original.

LELL: Metodología, supervición, validación, redacción: revisión y edición.

Fuentes de financiamiento

Autofinanciado.

Conflictos de interés

No tiene ningún conflicto de intereses.

Correspondencia

gintriago8813@utm.edu.ec 